\section{Standardizing SEM Beam Current for EDS Without Use of a Current Meter.}

Gib Ahlstand, University of Minnesota

Minnesota Agricultural Experiment Station

If you do not have a Faraday-type incident beam current meter on your SEM, use the following procedure to set up a calibrated, reproducible beam current when doing EDS. Of course, you won't know the actual value of the beam current in picoamps, but at least you'll know you can set the same value from sample to sample and day to day.

Mount a small, clean metal foil, $2 \times 3 \mathrm{~mm}$, aluminum or copper, onto the edge of each stub that the samples and standards are mounted on using colloidal graphite paint or double stick tape (after carbon or other evaporative conductive coatings have been applied to sample). Run a little colloidal graphite around an edge or two to provide additional adhesive support and a conductive bridge between the foil and the stub. Before taking sample or standards spectra, move the electron beam to the foil and as you accumulate its spectrum, set a region of interest, or window, on the princlple K-shell peak of the aluminum or copper spectrum. Look at the metal foil count rate on your EDS and adjust the beam current using the condensor lens, or spot size, control to set an arbitrary rate, say 3,000 counts per second, near the high end of $x$-ray through-put for your system (especially for biological samples - you may not need a high end setting for materials samples), but not more than, say, $33 \%$ deadtime. This value should be checked for stability periodically and should be checked for each new stub you put into the SEM. Thus you will be delivering the same incident beam current to your unknown samples. Of course, there is always some indeterminate error with any kind of instrumental setting, and the count rate fluctuates a bit from second to second due to detection and counting statistics in the electronics and the physics of beam current production. With our set-up, this fluctuation is about 100 counts or less from second to second around the desired level of 3,000 counts and over a 100 second analysis time these fluctuations average out. Also, our SEM (a Philips
$500 X)$ is quite stable. Provided the filament doesn't shift position, the same 3,000 cps will be there a few hours later. We set the rate when we put a sample in, and check it about every 30 minutes to monitor any changes that may occur. If a change has occured, we must throw out some data and collect over again.

A few precautions: 1) Use only very clean foils (prepare in advance, sonicate in mild detergent, rinse with distilled water, then ethanol, dry, store in covered Petri dish); 2) Actually image the foil surface at moderate magnification to make sure that you place the beam on a smooth area without microscratches; 3) Empirically determine, then record and consistently use standard $x$-ray excitation and collection parameters: $K v$, emission, stage tilt detector-to-foil target distance, working distance, count rate monitoring window width and centroid postion, EDS time constant and check energy axis calibration before beginning. Whew!

If your SEM beam current is often unstable over a few minutes time, you will have to check the foil count rate immediately before and after collecting a spectrum and keep only those spectra where the rate did not change during the analysis time. If it does change frequently, you may only be able to do limited standardless quantitative analysis or relative analysis based on ratios to one element in the spectrum.

One suggestion regarding the possible use of a specimen current meter to set incident beam current (I've not actually done this!): You can't do it by monitoring the absorbed current on the actual unknown specimen, because of varying current conductivity and backscattered electron reflectivity from region to region of sample. However, if the absorbed specimen current is measured on a foil (low Z prefered in this case) mounted as described above (important that foil be well grounded to stub), it would seem that a reproducible value can be set. This value is the total incident current minus the backscattered fraction, and this fraction should stay the same as long as you use the same type of foil target, $\mathrm{kV}$, geometry, etc, etc, etc, etc. You are sampling the same fraction of the total and are therby standardizing the total incident current. (of course, if you HAVE a specimen current meter, why not go one step further and mount a Faraday cup collector on the stage just beyound the edge of the sample stub and measure the incident current directly).
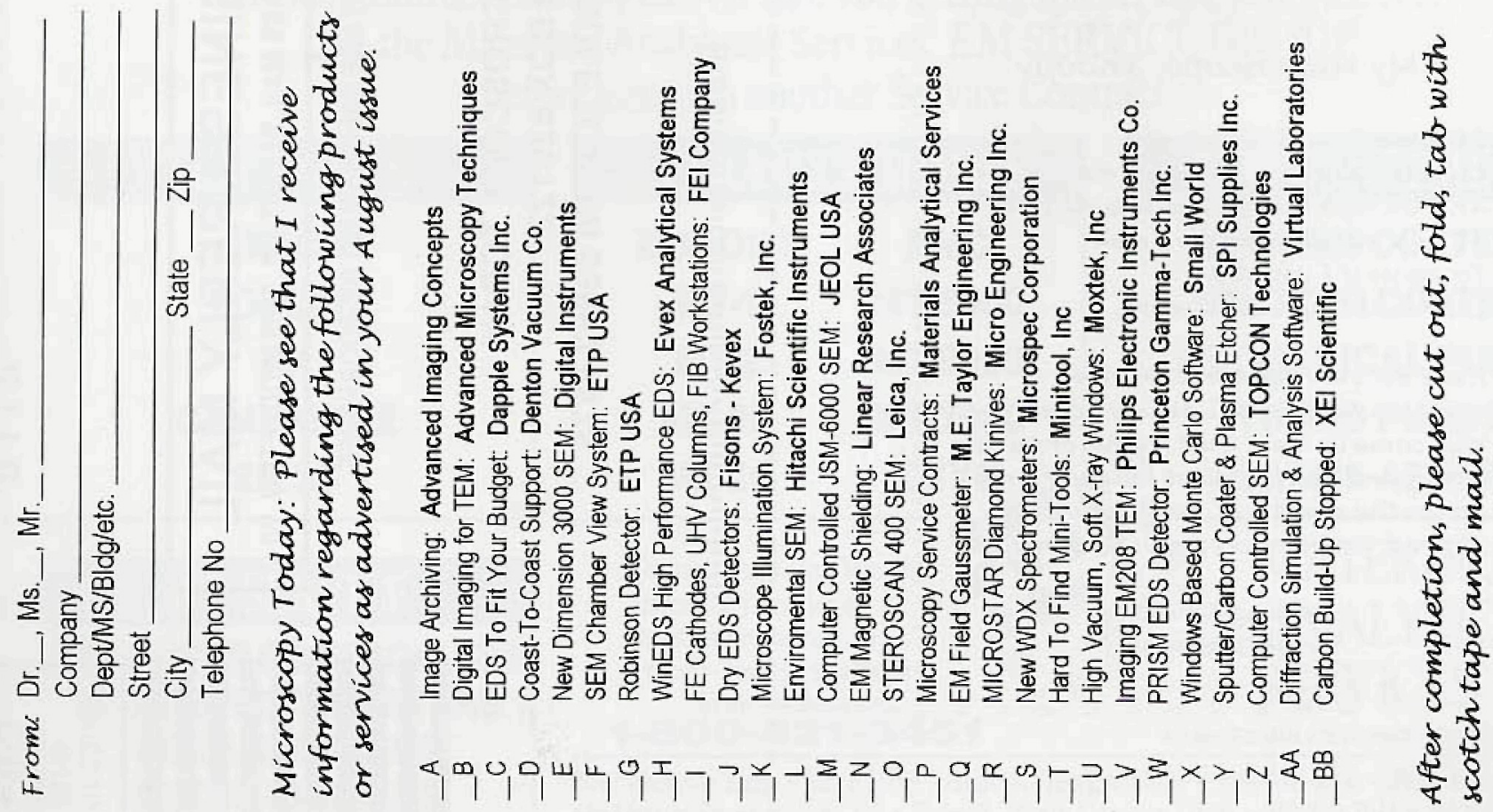